\title{
The importance of studying active giant stars in eclipsing binaries - and the role of citizen scientists in finding them
}

\author{
K. Oláh ${ }^{1}$, S. Rappaport ${ }^{2}$, A. Derekas ${ }^{3}$, A. Vanderburg ${ }^{4}$ and Citizen \\ Scientists: Tom Jacobs, Daryll LaCourse, Martti Kristiansen, Hans \\ Martin Schwengeler, Ivan Terentev \\ ${ }^{1}$ Konkoly Observatory, CsFK, Konkoly Thege M. u. 15/17, 1121 Budapest, \\ Hungary \\ 2 Department of Physics, and Kavli Institute for Astrophysics and Space \\ Research, Massachusetts Institute of Technology, Cambridge, MA 02139, \\ USA \\ ${ }^{3}$ ELTE Eötvös Loránd University, Gothard Astrophysical Observatory, 9700, \\ Szombathely, Szent Imre herceg u. 112., Hungary \\ 4 Department of Astronomy, The University of Texas at Austin, Austin, TX \\ 78712, USA, NASA Sagan Fellow
}

Received: October 30, 2019; Accepted: November 11, 2019

\begin{abstract}
Red giant stars with deep convection zones and rapid rotation maintain a strong surface magnetic field which may alter their observable astrophysical parameters. The resulting lower surface temperature due to spots makes the inferred masses and ages from evolutionary tracks uncertain. Eclipsing binaries having an active giant component can help in finding the stellar mass independently.

However, until the recent space missions it was nearly impossible to find such systems from the ground. Since the evolution on the giant branch is rapid, the number of binaries containing giant stars is low. The eclipses, if the inclination allows, are very shallow, on the order of the photometric accuracy from the ground, due to the large brightness difference between a red giant primary and its solar size or smaller secondary. And, the typically acquired data from the ground are not uniform or continuous.

In this paper, a few new eclipsing binaries are presented with active giant components observed by TESS and discovered by citizen scientists, which are worthy of further studies.
\end{abstract}

Key words: active stars - eclipsing binaries - space photometry

\section{Introduction}

Red giant stars in close binary systems often show signs of magnetic activity, like spots observable as rotational modulation, and flares (for example see Oláh 
et al. 2013). Some of these kinds of stars have huge amplitude variability both on rotational and long-term time-scales that span several tenths to about one magnitude in range. The photometrically observed stellar parameters thus also vary, and do not fit the theoretical evolutionary tracks corresponding their estimated mass (for example, see three such cases in Oláh et al., 2014). Studying active giants in eclipsing binaries can help to better constrain the stellar parameters, and through this the effect of the strong magnetic field on the stellar evolution. The first example of such an investigation which already involved citizen scientists has been published last year on EPIC 211759736 by Oláh et al. (2018).

\section{Data and Methods}

The depths of eclipses in a system containing a red giant primary and a solar size or smaller secondary is shallow and last for several hours to even days. The ground based observations are not continuous since one or at most a few datapoints are obtained on clear nights, and the data accuracy is similar to the depths of the eclipses. Data from space missions (Kepler, TESS satellites) overcome these drawbacks providing very accurate uninterrupted datasets with high time-resolution.

Visual surveyors of space-based light curves are very good at finding stars with variable light curves and shallow eclipses. Automated searches using BLS (box least squares) techniques can find them as well, but to our knowledge no one is pursuing this approach. The group of citizen scientists in the authorlist (and many more) spend a lot of their free time in searching for interesting objects in the databases. In the following a few intersting examples of their discoveries from the TESS database (Ricker et al., 2015) are presented, reduced from FFI images by FITSH (Pál, 2012).

\section{Results}

The main result of this survey is a (growing) list of potentially interesting active giant stars in eclipsing binaries. Detailed studies of satellite data supported with additional radial velocity measurements and multicolor photometry would allow us to get absolute stellar parameters. Table 1 lists five eclipsing binaries with active primaries, and their basic physical parameters as given in the GAIA DR2 catalog. These systems were found by citizen scientists.

The ground based observations to acquire radial velocity curve and starspot temperature from multicolor photometry are already ongoing to support a detailed study of TIC 375144608 .

TIC 123153249 (Fig. 1) is a known active star in a binary system, V344 Pup. This star has published radial velocity data and multicolor $\left(U B V R_{C} I_{C}\right)$ light curves dating back decades. It has never been discernible that the binary has 
Table 1. Sample of interesting red giants in eclipsing binaries from TESS data. Physical parameters are from GAIA DR2 catalog, orbital periods are own estimates.

\begin{tabular}{llllll}
\hline TIC & $\begin{array}{l}\mathrm{P}_{\text {orb }} \\
\text { days }\end{array}$ & $\begin{array}{l}\mathrm{T}_{\text {eff }} \\
\mathrm{K}\end{array}$ & $\begin{array}{l}\text { radius } \\
\mathrm{R}_{\odot}\end{array}$ & $\begin{array}{l}\text { lum. } \\
\mathrm{L}_{\odot}\end{array}$ & $\begin{array}{l}\text { TESS } \\
\text { mag. }\end{array}$ \\
\hline 123153249 & 11.761 & 4780 & 7.73 & 28.07 & 5.924 \\
271892852 & 4.150 & 4870 & 2.04 & 2.07 & 12.452 \\
375144608 & 13.4893 & 4680 & 8.55 & 36.16 & 9.959 \\
166974938 & 7.475 & 5230 & 5.03 & 14.11 & 11.310 \\
220461013 & 50.8 & 4550 & 6.52 & 16.95 & 10.662 \\
\hline \hline
\end{tabular}

eclipses: they are too shallow to be noticed from the ground. In Fig. 1, left, the TESS long- and short, cadence light curves are presented while on the right an old $\mathrm{V}$ dataset is shown. The estimate of the inclination from the old data was about $45^{\circ}$ (Stawikowski \& Glebocki, 1994), while, concerning the eclipses, the true inclination is around $75-80^{\circ}$, which results in a significant difference in the derived stellar parameters.
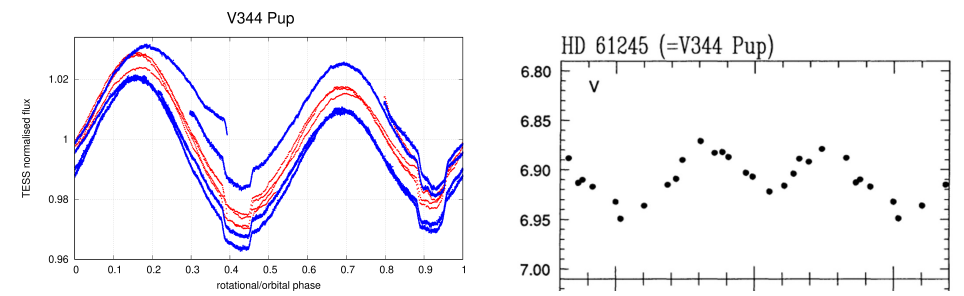

Figure 1. Left: TIC $123153249=$ V344 Pup observed parallel in long cadence (blue) and short cadence (red, from the MAST site, https://mast.stsci.edu) modes. The shifts between the light curves are not real. Right: Detail of an old multicolor dataset from Cutispoto (1991), the deepest datapoint may reflect the eclipse.

TIC 271892852, plotted in Fig. 2, left panel, is observed in all TESS sectors 1-13. It shows slow but marked changes in its light curve presumably as its starspots evolve over time, and at least one big flare. The spots affect the shape of the eclipses, and their depths are $0.06 \mathrm{mag}$. and $0.02 \mathrm{mag}$. No radial velocity measurements exist for this very interesting object.

TIC 166974938, plotted in Fig. 2, middle, is observed in TESS sectors 1, 3-11, and 13. Its light curve is slowly evolving in time, and large flares occur. Both eclipse depths are on the order of $0.01 \mathrm{mag}$. The secondary eclipse shows an interesting variability pattern. No radial velocity data is available for the system. 

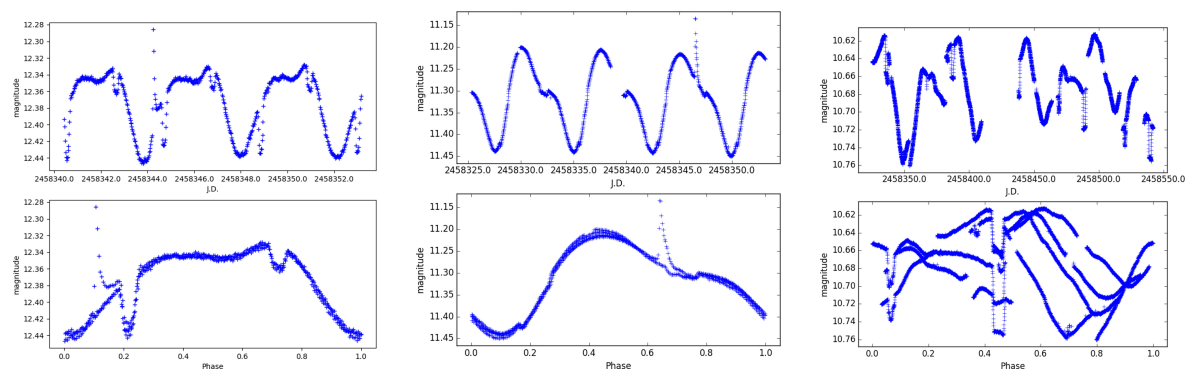

Figure 2. From left to right: example light curves of TIC 271892852, TIC 166974938 and TIC 220461013.

TIC 220461013, plotted in Fig. 2, right panel, is observed in TESS sectors 1-3 and 5-13. The star has longer orbital and rotational periods than the sector length of TESS. The rotational period of the giant star is definitely longer than the orbital period (about 54.9 days), and the orbit is eccentric. It is a possible triple system. No radial velocity data have been acquired.

All listed stars in Table 1 deserve further observations and modeling.

Acknowledgements. Thanks are due to Tamas Borkovits for inspiration and help. We acknowledge the use of public data collected by the TESS mission. This work has made use of data from the European Space Agency (ESA) mission Gaia, processed by the Gaia Data Processing and Analysis Consortium (DPAC). Funding for the DPAC has been provided by national institutions, in particular the institutions participating in the Gaia Multilateral Agreement.

\section{References}

Cutispoto, G., UBVRI observations of southern hemisphere active stars. II. 1987 data. 1991, Astron. Astrophys., Suppl., 89, 435

Oláh, K., Moór, A., Kővári, Z., et al., Magnitude-range brightness variations of overactive K giants. 2014, Astron. Astrophys., 572, A94, DOI: 10.1051/0004$6361 / 201424695$

Oláh, K., Moór, A., Strassmeier, K. G., Borkovits, T., \& Granzer, T., Long-term photometry of three active red giants in close binary systems: V2253 Oph, IT Com and IS Vir. 2013, Astronomische Nachrichten, 334, 625, DOI: 10.1002/asna.201211846

Oláh, K., Rappaport, S., Borkovits, T., et al., Eclipsing spotted giant star with K2 and historical photometry. 2018, Astron. Astrophys., 620, A189, DOI: 10.1051/0004$6361 / 201834106$

Pál, A., FITSH- a software package for image processing. 2012, Mon. Not. R. Astron. Soc., 421, 1825, DOI: 10.1111/j.1365-2966.2011.19813.x 
Ricker, G. R., Winn, J. N., Vanderspek, R., et al., Transiting Exoplanet Survey Satellite (TESS). 2015, Journal of Astronomical Telescopes, Instruments, and Systems, 1, 014003, DOI: 10.1117/1.JATIS.1.1.014003

Stawikowski, A. \& Glebocki, R., Are the Rotational Axes Perpendicular to the Orbital Planes in the Binary Systems? II. Synchronous Long-Period RS CVn Stars. 1994, Acta Astronomica, 44, 393 